\title{
What Is Time? Why Does Nature Love to Hide? How Does Object Influence Time? How Psychology Interacts with the Cycles of Time of Reference Which is Ideal Cycles of Time
}

\author{
Prasenjit Debnath \\ PhD Student, NIT Agartala, India
}

\begin{abstract}
What is time? This very deceptive simple question is the lone most important problem facing science as we go more deep into the fundamentals of the Universe - which is popularly known as crisis in physics or science. All the mysteries physicists and cosmologists face- from the Big Bang theory to the future of the Universe, from the quantum mechanics to the futuristic the unification of physics- all come down to the nature of time. The advancement of science puts milestones by dismissal of illusions. One of the key achievements is the hugely diversified Universe made of very simple rule and element i.e. everything in the Universe is actually made of different combination of protons, neutrons and electrons which are revolving around the nucleus. The protons and neutrons are made of still more elementary particles called quarks. The time is the most pervasive aspect of our everyday experience. We perceive the world as a flow of temporal moments that make up our life. Till date, to the physicists, philosophers and cosmologists, time is the ultimate illusion. Is that everything time bound? It is true that we are time bound and everything else in the world, that we are interact with, are all time bound. But there can be things that are timeless in nature. It is also true that we do not interact with timeless objects because till date, we never found any; whatever we found, all are time bound objects. Is it our inability to do that or is it a property of time bound objects? For example, truth, the truth is one and unique. The shape of truth is not a function of time, in other words, the truth is timeless, and that is why sometime it is popularly known as the ultimate truth to show that it is absolute in nature. For everything in the Universe, there is a timeless ultimate absolute truth exists, although truth is psychologically relative to us because we never know the ultimate truth which is absolute. For example, what is the ultimate elemental structure of the Universe? The answer can be only one which is the ultimate truth of this question; we do not know the answer, but we know one property of it, the elemental structure of the Universe is amazingly simple. It might be possible that there is only one elementary particle responsible for the hugely diversified Universe, but still we do not know the ultimate truth about the elemental structure of the Universe yet. There can be many questions which are timeless in nature. Like, for example, what is the reason for space existence? What is the relationship of time with space? We do not know the answers but we know that there is amazing regularity among elemental space points, all looks the same. So is true with elemental time associated with all elemental space points. Is that an accident that nature hides the timeless ultimate truth from us? Is it the ultimate truth that time gets reborn at next discrete time instant by demolishing the previous time instant? In other words, the time gets rebirth at every time instant and all time bound things accompany or interact with unidirectional making and breaking of time instants within the time itself, thus, all time bound things get rebirth at every time instant as time instant itself takes birth to form next time instant by demolishing the previous time instant. That is the reason we cannot penetrate past or future because they are purely psychological. It is the only discrete time instant that exists is the present time instant. The future-time instant is yet to form to go to the future by demolishing the present time instant. Past time instants are no more exist to be penetrated through. Because the ultimate truth is timeless, human beings cannot interact with truth as we are time bound. This is the reason why the truths of the Universe are always mystery for us. It proves that time bound thing can never interact with timeless thing; on psychological point of view, nature loves to hide.
\end{abstract}

Keywords: The fundamentals of the Universe, Crisis in physics or science, time bound objects, time reborn, discrete time instant.

\section{Introduction and Theory}

It seems that, the mathematical objects are constituted of pure thought $[1,2]$ - they are not part of the real world [3, 4]. But they are good idealistic model of the many phenomenon of the real world $[5,6]$. In that sense, the mathematical objects are timeless [7, 8]. For example - a circle is a set of points equidistant from a single point $[9,10]$ and a parabola is a set of points equidistant from a point and a line $[11,12]$. Amazingly, the path of the all falling objects on the Earth is always a parabola [13, 14]; the particle, whether thrown or dropped, falls with a constant acceleration $[15,16]$ although parabola came in mathematics way before that the observation of real particle falling on Earth[ 17, 18]. Falling is Universal [19] and a timeless truthso is the kind of curve that falling bodies trace [20, 21]. It does not matter what the object is made of, how it is put together or what its function is $[1,22]$. Nor does it matter how many times, from what height, or with what forward speed we drop or throw the object $[23,24]$. We can repeat the experiment thousand times and each time it's a parabola $[25,26]$. A parabola is one of the simplest curves to describe [27]. There can be numerous example of timeless truth, like for example, god exists or god does not exist. Only one option can be correct, which one is correct is the ultimate timeless truth and we do not know the answer. If there is no proof that god exists, then also there is no proof that he does not exist which in turn proofs that nature always loves to hide [28, 29]. If the truth is timeless for physicists, it is eternity for religious people [30]. The time bound and timeless difference made it impossible for human being to find the ultimate truth, in other words, nature will never reveal its mystery to human beings. 


\section{How Does Object Influence Time?}

Although there is nothing called free space, still if we consider a space that is very very far from all objects like planets or stars as the frame of reference and term as free space, then time flow can be modeled as minimum deviation from straight line in the so called free space. It may not be possible to get time curvature as a straight line as we cannot get pure free space with any external influence such as masses and energies.

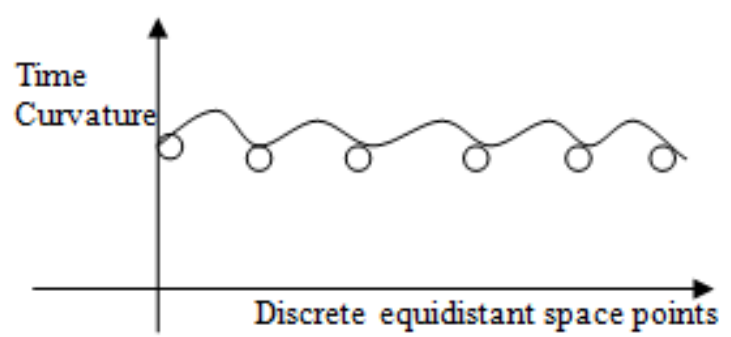

Figure 1: Because the time curvature is less curved, to keep the same unit vector length of time between space points, the horizontal component of time is large (real part of time- $\mathrm{x}$ axis component is large) - time runs fast in the free space.

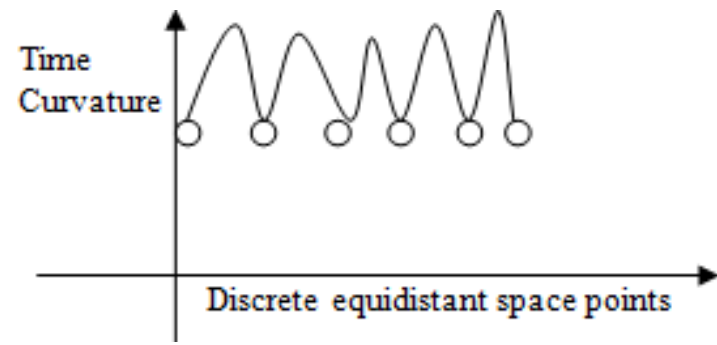

Figure 2: Because the time curvature is more curved to keep the same unit vector length of time between space points, as the space points are squeezed in objects (positive deformation of space points to create real objects of various form-solid, liquid and gas) the horizontal component of time is small (real part of time- $\mathrm{x}$-axis component is small) - time runs slow under the influence of real objects.

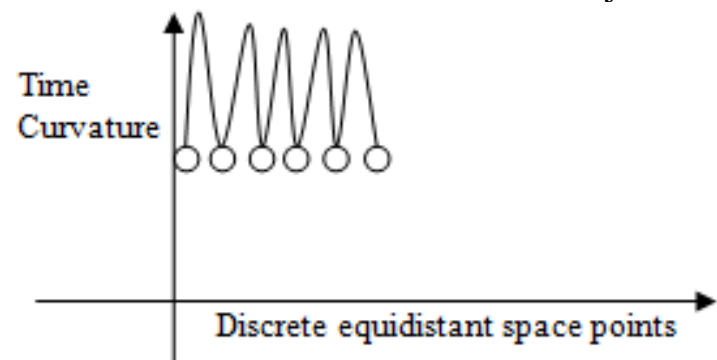

Figure 3: Because the time curvature is most curved to keep the same unit vector length of time between space points, as

the space points are most squeezed in the Black Holes (

highest positive deformation of space points to create real objects like Black Holes) the horizontal component of time is least possible (real part of time- $\mathrm{X}$-axis component is least possible) - time runs slowest possible under the influence of real objects like Black Holes.

\section{How Psychology interacts With the Cycles of Time of Reference- Which is Ideal Cycles of Time.}

Under normal state of mind, say happiness, the psychology interacts with every cycles of time available for an object like Earth.

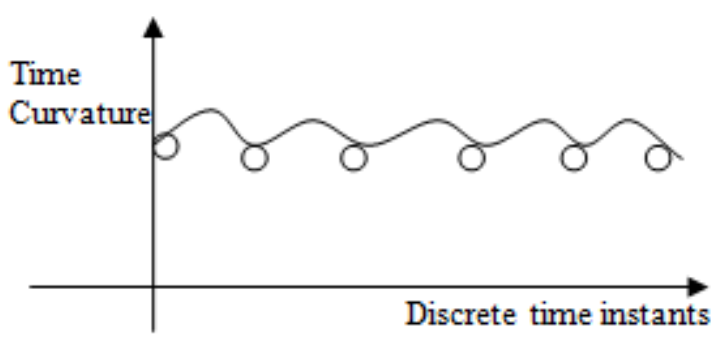

Figure 4: Psychology interacts with every time instants (Unidirectional).

If we feel suppression or depression time runs slower for us. The reason is that the psychology does not interact with every time instants available. The number of skipping of time instant is directly proportional to the degree of boredom.

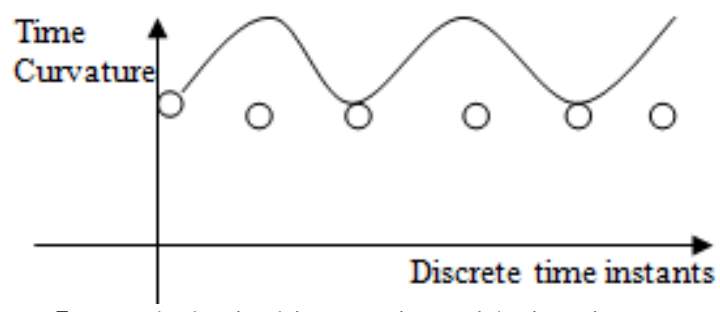

Figure 5: Psychological interaction with time instants under slight boredom (Unidirectional) - Every alternative time instant skipping - times runs little slower.

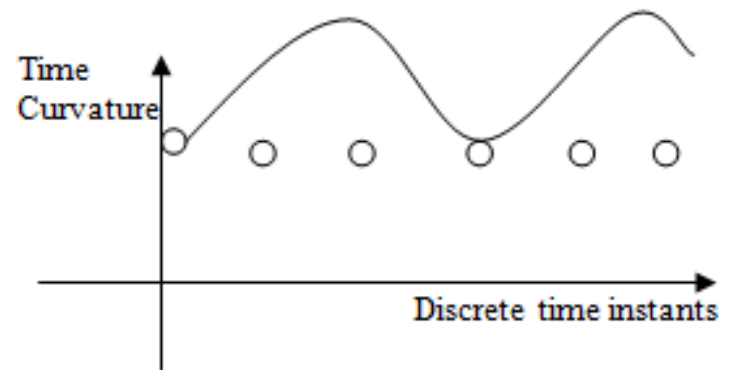

Figure 6: Psychological interaction with time instants under slightly higher degree of boredom (Unidirectional) - Two successive time instants skipping - time runs further slower.

\section{Conclusion}

What is time? It looks like a very simple question, but the reality is that it is the long term crisis in physics. Decoding the nature of time has become extremely essential to unify physics to have a complete consistent theory of physics that can be application to the entire Universe at a time. All physical theories are partial theories because of lack of knowledge about the time itself. Because the ultimate truth can reveal the mystery of the Universe, but because it is time less, it is impossible for us to reveal the ultimate truth as we are time bound creatures. Thus, nature just loves to hide 


\section{International Journal of Science and Research (IJSR) \\ ISSN (Online): 2319-7064 \\ Index Copernicus Value (2013): 6.14 | Impact Factor (2015): 6.391}

everything from us. Objects like Earth or Sun just slow down the temporal movement in the vicinity of these objects, highest slowness of unidirectional time flow can be achieved by the Universe in the vicinity of a Black Hole. Under normal condition psychology interacts with every time instants (which can be termed as ideal cycles of time), thus one can be free from the boredom in this process. But under adverse conditions psychology skips some time instants and interacts with some time instants, thus we feel boredom. The number of time instants skipping is directly proportional to the degree of boredom - time runs slower and slower according to the degree of boredom. We can never achieve the time less truth or the ultimate truth; the mystery of nature can never be completely revealed by human race- we have no option but to be happy with partial theories only.

\section{Acknowledgment}

I cordially admire Dr. Aparna Nath, Associate Professor and my PhD Guide, The department of Physics, National Institute of Technology, Agartala, India, for the epitome of inspiration and motivation to write this particular paper with perfection and accuracy. I am extremely thankful to her from all possible help she made to write this paper. Also I am thankful to The Department of Physics of National Institute Of Technology Agartala (NIT Agartala) for proper conduct and coordination.

\section{References}

[1] Lee Smolin, " Time Reborn: From the Crisis in Physics to the Future of the Universe" Penguin Books, pp. 1-41

[2] Prasenjit Debnath, "Time and Space Are Mutually Inherent Property of Each Other, Where the Dependency of Time on Space Can Be Termed as Space-Time and the Dependency of Space on Time Can Be Termed as Time-Space" International Journal Of Science And Research, ISSN (online)- 2319-7064, volume 5 Issue 2, pp 1946-1948

[3] Brian Cox, Jeff Forshaw, "The quantum universe: everything that can happen does happen", Penguin Books, pp. 1-44.

[4] Stephen Hawking, "A Briefer History of Time", Bantam Books, London, pp. 1-145.

[5] http://map.gsfc.nasa.gov/universe/uni_fate.html

[6] Stephen Hawking, "Black holes and Baby Universes and other essays", Bantam Press, London 2013, ISBN 978-0-553-40663-4

[7] Stephen Hawking, "The Grand Design", Bantam Books, London 2011

[8] Stephen Hawking, "A Brief History of Time", Bantam Books, London 2011, pp. 156-157. ISBN-978-0-55310953-5

[9] Stephen Hawking, "The Universe in a Nutshell", Bantam Press, London 2013, pp. 58-61, 63, 82-85, 90-94, 99, 196. ISBN 0-553-80202-X

[10] Stephen Hawking, "The Beginning of Time", A Lecture.

[11] Stephen Hawking, “Stephen Hawking's Universe: Strange Stuff Explained", PBS site on imaginary time.

[12] Stephen Hawking, "How to build a time machine", 27 April, 2010.
[13] Uno Ingard, K "Fundamental of Waves \& oscillations", Cambridge University Press. P. 38, ISBN-0-521-33957XOxford: The British Academy, 1999

[14]A. Zee, "Quantum Field Theory in a Nutshell", Princeton University Press, 2003

[15] Storrs McCall, "A Model of the Universe", Oxford: Clarendon Press, 1994

[16]Craig Callender, "Time, Reality and Experience", Cambridge, UK: Cambridge University Press.

[17]Craig Callender, "Thermodynamic Asymmetry in Time", The Stanford Encyclopedia of Philosophy (Spring 2002 Edition)

[18] Storrs McCall, "A Model of the Universe", Oxford: Clarendon Press, 1994

[19]Robin Le Poidevin and Murray McBeath, "The Philosophy of Time" Oxford: Oxford University Press, 1993

[20] Newton-Smith, W.H., "The Structure of Time". London: Routledge \& Kegan Paul, 1980.

[21]Barry Dainton,"Time and Space", Ithaca: McGillQueen's University Press, 2001

[22] Robin Le Poidevin, "Questions of Time and Tense", Oxford: Oxford University Press, 1998.

[23] Nerhlich, Graham, "What Spacetime Explains". Cambridge: Cambridge University Press, 1994.

[24] Sklar, Lawrence, "Space, Time, and Space-time". CA: University of California Press, 1974.

[25] Whitrow, G., "The Natural Philosophy of Time". Oxford: Oxford University Press, 1961. (2nd edn., 1980.)

[26] S.W. Hawking, and G.F.R. Ellis, "The Large Scale Structure of Space-Time", Cambridge University Press, (1973).

[27] Stephen Hawking, “A stubbornly persistent illusionThe essential scientific works of Albert Einstein", Running Press Book Publishers, Philadelphia, London 2011.

[28] Flynn, John L, "Time travel literature", on 29-09-2006

[29] Stephen Hawking, "The Theory of Everything", Jaico Books, pp. 1-110.

[30] Stephen Hawking, "The Illustrated A Brief History of Time", Bantam Books, pp. 1-21.

\section{Author Profile}

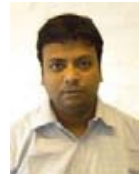

Prasenjit Debnath born in Agartala, Tripura, India on $15^{\text {th }}$ of March 1979. He is pursuing a PhD degree in the Department of Physics in National Institute of Technology Agartala (NIT Agartala), India. 